\title{
Effects of Narrow-band UVB Alone and in Combination with Isotretinoin on Apoptosis and Its Clinical Implications in Early Stage Mycosis Fungoides
}

\author{
(1) Nazan Tașlıdere' , (1) Özlem Canöz², (1) Ayten Ferahbaș Kesikoğlu³ \\ ${ }^{1}$ Bezmialem Vakif University, Department of Dermatology and Venereology, Istanbul, Turkey \\ 2Erciyes University Faculty of Medicine, Department of Pathology, Istanbul, Turkey \\ ${ }^{3}$ Group Florence Nightingale Hospitals, Clinic of Dermatology, Istanbul, Turkey
}

\section{ABSTRACT}

Background: Mycosis fungoides, one of the most common subtypes of cutaneous T-cell lymphomas, is characterized by uncontrolled T cell proliferation, apoptosis resistance, and consequent chronic cutaneous inflammation. This study aims to show the effects of narrow-band ultraviolet B (UVB) alone and narrow-band UVB + isotretinoin treatments on the expression of fas, fas ligand, Bcl-2, STAT-3 and galectin-3 proteins which are responsible for apoptosis regulation, and also assess the relationship between this regulatory functions and clinical improvement.

Materials and Methods: The first group received narrow-band UVB alone and the other group received narrow-band UVB combined with $0.5 \mathrm{mg} / \mathrm{kg} /$ day oral isotretinoin. After 30 sessions of treatment, biopsies from the patient groups were examined immunohistochemically for the expression of fas, fas ligand, Bcl-2, STAT-3 and galectin-3 protein.

Results: In the narrow-band UVB only group, the intensity of epidermal Bcl-2 staining was found to be increased in the post-treatment samples when compared to the pre-treatment. On the other hand, epidermal staining intensities and diffuseness for fas, fas-ligand, Bcl-2, STAT-3, and galectin-3, and also the intensities and diffuseness of epidermal lymphocyte staining and presence of epidermal cells were similar between pre and post-treatment samples within each group separately, and between the two treatment groups overall.

Conclusion: In the treatment of early stage mycosis fungoides, darband UVB and retinoic acid are used. In our study, the effects of these treatments on apoptosis could not be demonstrated. Larger series studies are needed in this regard.

Keywords: Mycosis fungoides, Narrow-band ultraviolet B, Isotretinoin

\section{Introduction}

Our skin is one of our largest organs, which we have the largest relationship with the external environment and constitutes approximately $16 \%$ of body weight [1]. While the epidermis exerts its barrier function mechanically, our skin plays an active role in the immune response to pathogens by the cellular elements it contains although it is not considered as a primary or secondary lymphoid organ. When the resident Langerhans cells are stimulated by proper signals, they turn into effective antigen presenting cells, causing an immune response against the target antigen under the control of $T$ and B lymphocytes [2]. Although the etiology of mycosis fungoides, one of the most common subtypes of cutaneous T-cell lymphomas, is not fully elucidated, genetic, environmental and infectious causes are accused. Monoclonal proliferation of T-cells in the epidermis 
and disruption of the apoptosis process which result from diffuse inflammatory reactions induced by chronic antigenic stimulation form the basis of the disease [3,4]. Uncontrolled T cell proliferation, resistance to apoptosis and consequent chronic cutaneous inflammation are responsible for the development of lymphoma. Primary cutaneous lymphomas have an overall incidence of 0.3-1.0 / 100.000 and 54-72\% of them are mycosis fungoides [5]. Clinically there are four stages of mycosis fungoides, namely patch, plaque, tumor, and erythroderma, and some cases may be characterized by atypical skin manifestations [6,7]. In the patch and plaque stage, lesions are often asymmetrical, itching is prominent and scalp involvement may result in alopecia $[8,9]$. These infiltrations, which begin as patches and plaques, progress into nodules and may show systemic spread when left untreated $[10,11]$. The gold standard for the diagnosis of mycosis fungoides is histopathological evaluation of skin biopsy. The treatment methods can be classified into three main approaches: methods targeting the skin, systemic treatment or biological agent regulators. The observation that the lesions frequently reside on the regions unexposed to sunlight led to the assumption that ultraviolet (UV) radiation may prevent the development of mycosis fungoides and thus PUVA, narrow-band and broadband UV B (UVB) treatments are used $[12,13]$. There are three types of retinoids: isotretinoin, acitretin and etretinate, which exert their effects via the retinoic acid receptor, and bexarotene which binds to the retinoid $\mathrm{X}$ receptor. All these retinoids show their therapeutic effects by inducing apoptosis [14]. The aim of this study is to investigate the effects of narrow-band UVB only and narrow-band UVB + isotretinoin treatments on the expression of fas, fas ligand, Bcl-2, bax, STAT-3 and galectin-3 proteins which are responsible for apoptosis regulation and the relationship between apoptosis regulation and clinical improvement.

\section{Materials and Methods}

This study was carried out between May 2006 and March 2010 at the Dermatology and Pathology Departments of the Faculty of Medicine and supported by the Scientific Research Project Commission of the University (project no: TT-06-34, approval no: 01/181). Twentyone patients diagnosed as mycosis fungoides patch stage clinically and histopathologically were included in the study after receiving consent form from patients. Demographic data of all patients were recorded. Dermatological examinations of the patients were performed. The total affected body area was calculated as $\mathrm{cm}^{2}$, and all treatments were ceased two weeks before skin biopsy. A total of two skin biopsies (i.e. pre-, and post-treatment) were obtained from the lesion area, and the diagnosis was confirmed by histopathology and immunophenotyping. Patients were randomly divided into two groups. Pre-, and post-treatment lesion size and symptom severity were evaluated. The first group was given narrow-band UVB treatment at 0.005 joules $/ \mathrm{cm}^{2}$ three days a week for a total of 30 sessions, increasing the applied dose by $30 \%$ in every third session. The second group received $0.5 \mathrm{mg} / \mathrm{kg} /$ day per-oral isotretinoin in combination with the narrow-band UVB treatment. After 30 sessions of narrow-band UVB treatment, each patient underwent a dermatological examination, in which clinical findings and symptoms were scored between 0 and 8 and lesion size between 0 and 18, and a cumulative score was calculated for each patient thereafter. Pre- and post-treatment biopsy specimens obtained from each patient in both study groups were initially examined with hematoxylin-eosin staining, followed by immunohistochemical evaluation for fas, fas-ligand, BCl-2, bax, galectin-3 and STAT-3 expressions in malignant lymphoid cells. For each patient, preand post-treatment images of the lesions were taken with a digital camera. Pre- and post-treatment data regarding clinical resolution and apoptosis were compared in and between both groups. Tissue samples were evaluated macroscopically and placed in a tissue processing system. Tissue samples were incubated in formaldehyde at $37{ }^{\circ} \mathrm{C}$ for 2 hours followed by embedding in alcohol solutions with increasing concentrations for 1 hour each. After 4.5 hours of incubation in xylol and 3 hours in paraffin, the samples were taken from the device and paraffin blocks were prepared. The blocks were refrigerated for 30 minutes before the preparation of 5 micrometer-thin sections on microscope slides containing poly-Llysine adhesive. The sections were incubated for 1 hour in an oven at $60^{\circ} \mathrm{C}$, processed with consequent xylol and alcohol solutions with decreasing concentrations, and finally rinsed with distilled water. Sections were then boiled for 20 minutes in 10\% citrate buffer solution. In order to minimize non-specific staining and background noise, all preparations were treated with $3 \% \mathrm{H}_{2} \mathrm{O}_{2}$ for 10 minutes and washed with buffered saline solution for 10 minutes. Readyto-use antibodies against fas, fas-ligand, bax, $\mathrm{Bcl}-2$, galectin-3 and STAT-3 were used as primary antibodies. Immunohistochemical staining was performed. The slides were treated with increasing concentrations of alcohol solutions, embedded in xylol and closed with a sealing solution. Intestinal tissue, prostatic tissue, Hodgkin lymphoma specimens, tonsillar tissue, thyroid tissue and breast cancer specimens were used as positive controls for fas, fas-ligand, bax, Bcl-2, galectin-3 and STAT-3 expression studies, respectively. Tissue preparations were examined under a light microscope. Immunohistochemically, cytoplasmic staining of keratinocytes and lymphocytes for bax and $\mathrm{BCl}-2$ was considered positive Unstained cells were deemed negative (Figure 1, 2, 3). For Bcl-2 and bax assessment, all microscopic fields were examined for pre- and posttreatment specimens. A total of 10 positively stained fields were examined under low power magnification and scored according to the average positively stained cell numbers as: score $0 \%(-), 1-25 \%$ $(+), 26-50 \%(++), 51-100 \%(+++)$. Staining intensities of lymphocytes 
were classified as no staining, weak, moderate and intense. Presence of epidermal lymphocytes before and after treatment was evaluated as no epidermal lymphocyte, few and present.

\section{Statistical Analysis}

Chi-square and Mann-Whitney $U$ tests were used to compare age, sex, and disease duration. Fas, fas-ligand, bax, BCl-2, STAT-3 and galectin-3 staining intensities before and after treatment were compared with Wilcoxon signed-rank test. Mann-Whitney $U$ test was used to compare the staining intensities of narrow-band UVB only and isotretinoin + narrow-band UVB treatment groups. In both treatment groups, intragroup pre- and post-treatment symptom severity and lesion size comparisons were performed using Wilcoxon signed-ranks test. Values of $p<0.05$ were considered statistically significant.
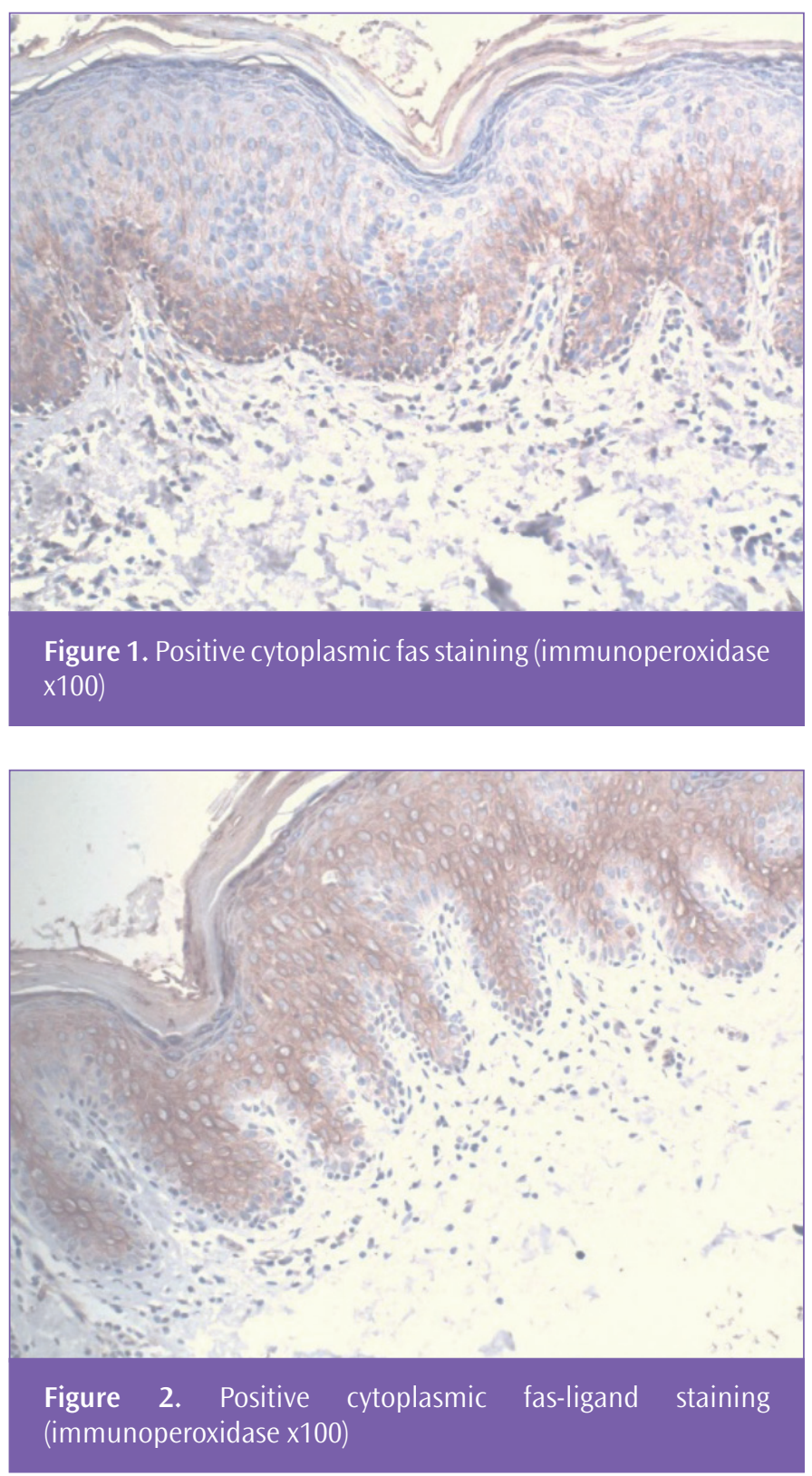

\section{Results}

In our study, 10 patients received narrow-band UVB alone and eleven patients received narrow-band UVB + isotretinoin combination treatment. Nine (42.9\%) of the patients were female and 12 (57.1\%) were male. Mean age was $48.33 \pm 12.39$ years in all patients, $50.00 \pm 12.15$ years in the narrow-band UVB only group, and $46.81 \pm 12.99$ years in the narrow-band UVB + isotretinoin group. There was no statistically significant difference between the groups in terms of gender and age ( $p=0.130, p=0.672$, respectively), (Table 1). Mean disease duration was $8.89 \pm 7.28$ years in the first group and $4.60 \pm 3.93$ years in the second group $(p=0.216)$. Eight of the patients were in stage $1 \mathrm{~A}, 9$ in stage $1 \mathrm{~B}$, and 4 in stage $2 \mathrm{~A}$. Six of the stage IA patients had narrow-band UVB only and 2 of them had narrow-band UVB + isotretinoin, 2 of the stage 1B patients had narrow-band UVB only and 7 had narrow-band UVB + isotretinoin, and 2 of stage $2 A$ patients had narrow-band UVB only and 2 received narrow-band UVB + isotretinoin treatment. Pre and post-treatment symptom severity scores were $12.50 \pm 6.16$ and $5.40 \pm 4.97$ in the narrow-band UVB only group and $16.90 \pm 4.50$ and $5.18 \pm 4.42$ in the narrow-band UVB + isotretinoin combination group, respectively. In both groups, the differences between pre- and post-treatment symptom severity scores were statistically significant $(p<0.009$ in narrow-band UVB only group and $p<0.003$ in narrow-band UVB + isotretinoin combination group). Overall, pre-treatment symptom severity was high and there was a significant decrease after treatment ( $\mathrm{p}=0.09$ ) (Table 2). In the first group, pre- and posttreatment lesion sizes were $10.80 \pm 6.01$ and $6.70 \pm 6.54$, while in the second group they were measured to be $14.63 \pm 2.54$ and $7.90 \pm 6.04$ (Table 3). The pre-treatment lesions in both groups were large, but there was a significant decrease in lesion sizes after the treatment. No significant difference was detected when pre-treatment lesion

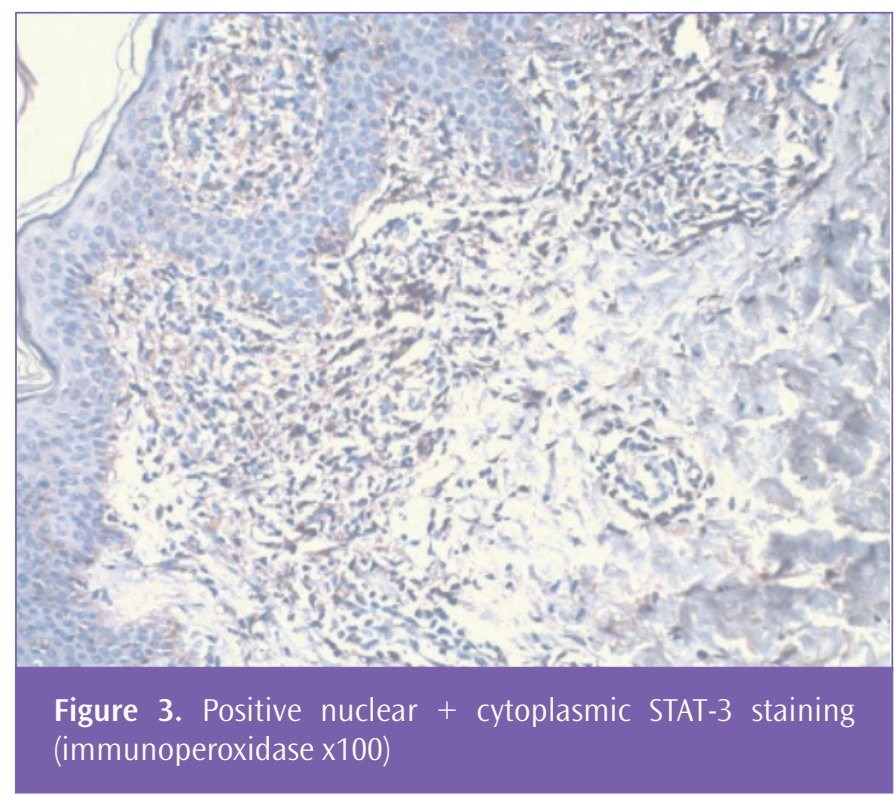


sizes and post-treatment lesion sizes were separately compared between the two treatment groups ( $p=0.20$ for pre-treatment sizes, $p=0.43$ for post-treatment sizes). Clinical response to treatment was significantly earlier in the narrow-band UVB + isotretinoin combination group than the narrow-band UVB only group, and the results were statistically significant. In the narrow-band UVB only group, the difference between the of $\mathrm{BCl}-2$ epidermal staining intensities obtained from pre- and post-treatment biopsy samples were statistically significant $(p=0.04)$. However in the same group, pre- and post-treatment samples revealed similar results regarding $\mathrm{Bcl}-2$ epidermal staining diffuseness, epidermal Iymphocyte staining intensities and diffuseness and presence of epidermal lymphocytes $(p>0.05)$. In addition, again in the same group, pretreatment and post-treatment epidermal staining intensities and diffuseness for fas, fas-ligand, bax, STAT-3 and galectin-3, as well as epidermal lymphocyte staining intensities and diffusiveness and presence of epidermal lymphocytes were not significantly different. In the narrow-band UVB + isotretinoin group, on the other hand, the intensity and diffuseness of epidermal staining for fas, fas-ligand, bax, Bcl-2, STAT-3 and galectin-3, the staining intensities and extent of epidermal lymphocytes and the presence of epidermal lymphocytes were not significantly different between the pre-treatment and post-treatment samples. Overall epidermal staining intensities and diffuseness for fas, fas-ligand, Bcl-2, STAT-
3 and galectin-3, epidermal lymphocyte staining intensities and diffuseness, and presence of epidermal lymphocytes did not show significant differences between the pre-and post-treatment samples $(p>0.05)$. No positive staining for bax protein was detected (Table 4).

\section{Discussion}

Mycosis fungoides is a cutaneous T-cell lymphoma characterized by varying degrees of atypical lymphocyte infiltration in the papillary dermis. Phototherapy is accepted as an effective treatment approach for mycosis fungoides in that it exerts a suppressive effect against T-lymphocytes and Langerhans cells. Although narrow-band UVB phototherapy is frequently used especially in the treatment of early stage mycosis fungoides, in our study it did not result in significant changes in apoptotic markers. This may be due to the fact that apoptosis is not yet a prominent process in the early stage of the disease. In advanced stages of mycosis fungoides, other systemic treatment alternatives may be combined with phototherapy. There are many alternative drugs such as isotretinoin, bexarotene and vorinostat. Retinoids are vitamin A derivatives that regulate cell proliferation, differentiation and apoptosis. Isotretinoin, one of the first retinoids, was observed to show its clinical effects earlier when combined with narrow-band UVB, rather than being used alone. However, there was no difference between pre-treatment and posttreatment results in our narrow-band UVB only and isotretinoin +

Table 1. Comparison of patient and control groups

\begin{tabular}{|l|l|l|l|l|}
\hline & Patient group & Narrow-band UVB & Narrow-band UVB + isotretinoin & $\mathbf{p}$ \\
\hline Number of patients & 21 & 10 & 11 & 0.827 \\
\hline Male female & $12 / 9$ & $4 / 6$ & $8 / 3$ & 0.130 \\
\hline Age (years) & $48.33 \pm 12.39$ & $50.00 \pm 12.15$ & $46.81 \pm 12.99$ & 0.672 \\
\hline UVB: Ultraviolet B & \multicolumn{5}{|l}{} \\
\hline
\end{tabular}

Table 2. Comparison of symptom severity before and after treatment between groups

\begin{tabular}{|c|c|c|c|}
\hline & \multicolumn{2}{|l|}{$\begin{array}{l}\text { Symptom severity } \\
\text { Mean } \pm \text { SD }\end{array}$} & \multirow[t]{2}{*}{$\mathbf{p}$} \\
\hline & Before treatment & After treatment & \\
\hline Narrow-band UVB + isotretinoin & $16.90 \pm 4.50$ & $5.18 \pm 4.42$ & 0.003 \\
\hline $\mathrm{p}$ & 0.09 & 0.91 & \\
\hline
\end{tabular}

Table 3. Comparison of lesion size before and after treatment between groups

\begin{tabular}{|l|l|l|l|}
\hline \multirow{2}{*}{} & \multicolumn{2}{|l|}{$\begin{array}{l}\text { Lesion size } \\
\text { Mean } \pm \text { SD }\end{array}$} & p \\
\cline { 2 - 4 } & Before treatment & After treatment & \\
\hline Narrow-band UVB & $10.80 \pm 6.01$ & $6.70 \pm 6.54$ & 0.007 \\
\hline Narrow-band UVB + isotretinoin & $14.63 \pm 2.54$ & $7.90 \pm 6.04$ & 0.012 \\
\hline$p$ & 0.20 & 0.43 & \\
\hline UVB: Ultraviolet B, SD: Standard deviation & & \\
\hline
\end{tabular}


Table 4. Before and after treatment; comparison of fas, fas-ligand, Bcl-2, STAT-3, galectin-3

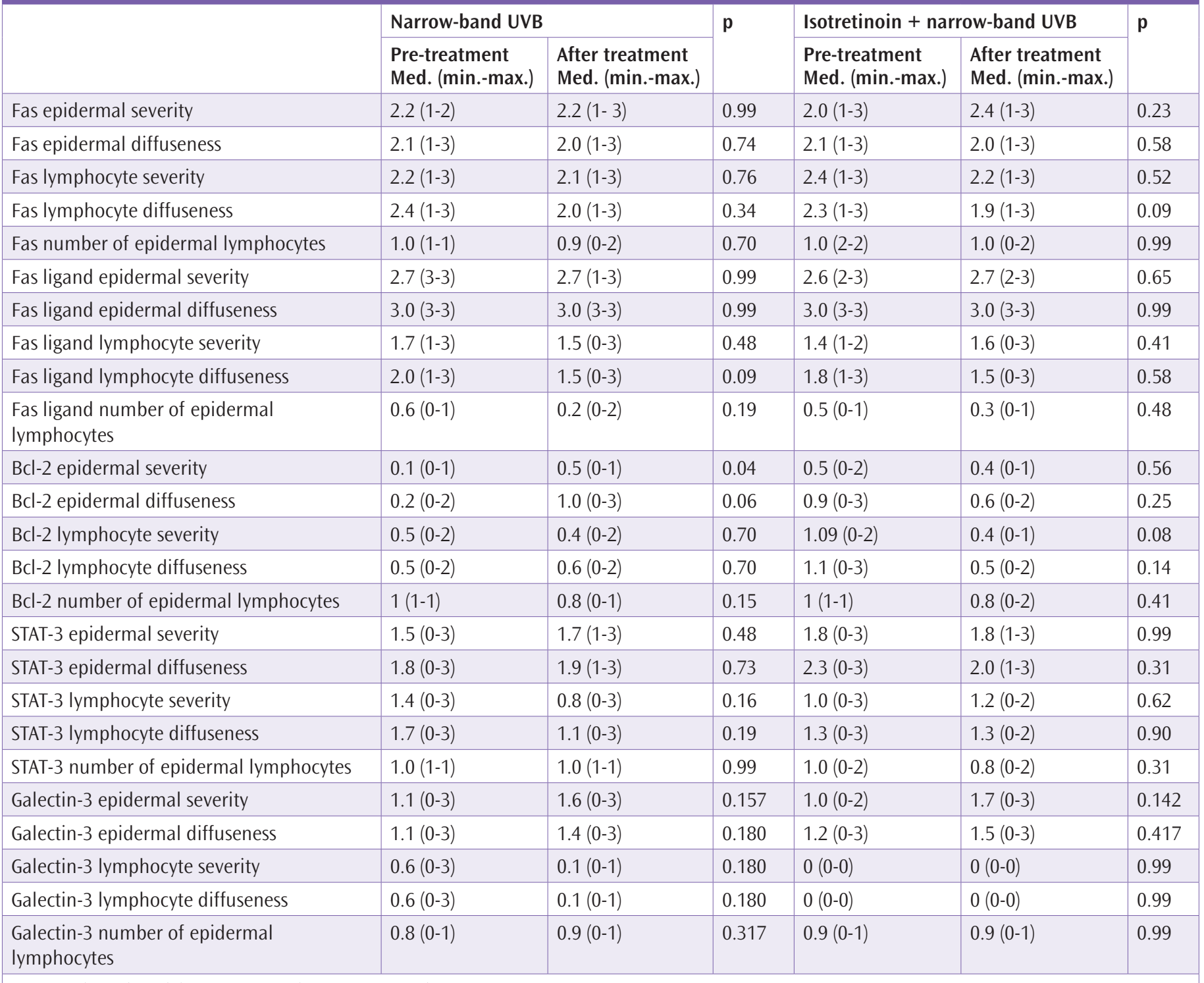

Med.: Median, min.: Minimum, max.: Maximum, UVB: Ultraviolet B

narrow-band UVB groups ( $p=0.005)$. Fas is an apoptosis-associated protein of lymphoid cells, and in mycosis fungoides, its expression decreases as the disease stage progresses. Narrow-band UV increases the expression of fas/fas-ligand, which has a strong inducing effect for apoptosis [15]. Fas-directed apoptosis is inhibited by the antiapoptotic $\mathrm{BCl}-2$ protein. The presence of $\mathrm{Bcl}-2$ expression leads to resistance development to apoptosis and is a negative prognostic factor. Our patients did not show a prominent Bcl-2 expression was observed in patients, and a number of previous studies have shown that $\mathrm{Bcl}-2$ expression is decreased in these patients [16], implying that $\mathrm{Bcl}-2$ function is not pronounced in early-stage patients. Proapoptotic bax protein induces apoptosis by increasing the production of fas and suppressing Bcl-2. Although increased bax expression following ultraviolet exposure and its role in intracellular signaling pathways in the pathogenesis of mycosis fungoides are well-established, bax levels were not found to be significant in our study [17]. STAT-3 activation was observed in malignant cells obtained from the skin and blood of patients with cutaneous T-cell lymphoma. STAT-3 positivity is rarely seen in early-stage mycosis fungoides lesions because most lymphocytes in this stage are inflammatory rather than neoplastic. We found that both cutaneous T-cell lymphoma patients and the control group showed positive keratinocyte staining for STAT-3 in the keratinocytes, revealing no significant difference between the patients and the control group [18].

STAT-3 positivity is an indicator of progression to advanced stages and this protein is one of the potential molecules that can be targeted in novel treatment strategies for patients in this stage. 
In our study, STAT-3 expression levels were similar between preand post-treatment samples in both groups, and between the two treatments groups. This may be attributable to the fact that STAT-3 expression is mostly observed in advanced stage patients and is not specific for malignant cells since STAT-3 is also present in proliferating keratinocytes. Galectin-3 is a lectin-binding betagalactosidase and its expression is increased in various neoplastic cell types. It is functionally involved in epithelial cell proliferation, malignant transformation and metastasis. Galectin-3 expressing cells show resistance to apoptosis [19]. In our study, the median post-treatment galectin-3 staining levels were increased when compared to pre-treatment levels in both groups, the differences being statistically insignificant. This was thought to be due to the variations in cell surface glycosylation between individuals.

\section{Study Limitations}

Difficulty in immunohistochemical detection of the apoptotic markers due to their short half-life, different skin types, interperson variability in retinoid receptor expressions, functional polymorphisms in retinoid metabolism.

\section{Conclusion}

According to our findings, narrow-band UVB + isotretinoin combination has no superiority to narrow-band UVB only treatment in terms of both clinical improvement and induction of apoptosis. However, recovery started earlier in the isotretinoin + narrowband UVB group. The results we obtained with our study revealed important issues that may establish a ground for further studies.

\section{Ethics}

Ethics Committee Approval: The study was Dermatology and Pathology Departments of the Faculty of Medicine and approved by the Scientific Research Project Commission of the University (project no: TT-06-34, Ethics Committee approval no: 01/181).

Informed Consent: Consent form was filled out by all participants.

Peer-review: Internally peer-reviewed.

\section{Authorship Contributions}

Data Collection or Processing: N.T., A.F.K., Analysis or Interpretation: A.F.K., Ö.C., Literature Search: N.T., Writing: N.T.

Conflict of Interest: No conflict of interest was declared by the authors.

Financial Disclosure: The authors declared that this study received no financial support.

\section{References}

1. Rush $\mathrm{A}$, Muir M. Maintaining skin integrity in bariatric patients. $\mathrm{Br}$ J Community Nurs 2012;17:154, 156-9.
2. West, $\mathrm{HC}$, Bennett, CL. Redefining the Role of Langerhans Cells As Immune Regulators within the Skin. Front Immunol 2018;8:1941.

3. Latkowski JA, Heald P. Cutaneous T Cell Lymphomas. In: Freedberg IM, Eisen AZ, Wolff K, [eds]. Fitzpatrick's Dermatology in General Medicine, Vol 2, 6th ed. New York: Mc Graw Hill; 2003. p. 1537-1558.

4. Rezk Hassan GF, Marey K. Immunohistopathological Study of c-FLIP Protein in Mycosis Fungoides. Asian Pac J Cancer Prev 2017;18:2493-2499.

5. Cengiz FP, Emiroğlu N, Onsun N. Frequency and Risk Factors for Secondary Malignancies in Patients with Mycosis Fungoides. Turk J Haematol 2017;34:378-379.

6. Yamashita T, Abbade LPF, Marques MEA, Marques SA. Mycosis fungoides and Sezary syndrome: clinical, histopathological and immunohistochemical review and update. An Bras Dermatol 2012;87:817-828; quiz 829-30.

7. Ferahbas A. Mikozis Fungoides, klinik varyantları ve subtipleri. Turkiye Klinikleri J Dermatol 2007;17:242-251.

8. Sokolowska-Wojdylo M, Olek-Hrab K, Ruckemann-Dziurdzinska K. Primary cutaneous lymphomas: diagnosis and treatment. Postepy Dermatol Alergol 2015;32:368-383.

9. Olsen EA. Evaluation, Diagnosis, and Staging of Cutaneous Lymphoma. Dermatol Clin 2015;33:643-654.

10. Mahalingam M, Reddy VB. Mycosis Fungoides, Then and Now. Have We Travelled? Adv Anat Pathol 2015;22:376-383.

11. Talpur R, Bassett R, Duvic M. Prevalence and treatment of Staphylococcus aureus colonization in patients with mycosis fungoides and Sezary syndrome. Br J Dermatol 2008;159:105-112

12. Giberson M, Mourad A, Gniadecki R. Folliculotropism Does Not Affect Overall Survival in Mycosis Fungoides: Results from a Single-Center Cohort and MetaAnalysis. Dermatology 2017;233:320-325.

13. Talpur R, Sui D, Gangar P. Retrospective Analysis of Prognostic Factors in 187 Cases of Transformed Mycosis Fungoides. Clin Lymphoma Myeloma Leuk 2016;16:49-56.

14. Humme D, Nast A, Erdmann R, Vandersee S, Beyer M. Systematic review of combination therapies for mycosis fungoides. Cancer Treat Rev 2014;40:927933.

15. Beyer M, Möbs M, Humme D, Sterry W. Pathogenesis of Mycosis fungoides. J Dtsch Dermatol Ges 2011;9:594-598.

16. Weshahy H, Mahgoub D, El-Eishy N, El-Tawdy AM, Bassiouny DA, Hunter N, Hindawi A. BCl-2 expression in mycosis fungoides before and after PUVA therapy. Photodermatol Photoimmunol Photomed 2010;26:107-109.

17. Aydin F, Levent Y, Nilgun S, Pancar YE, Yasar TA. Implications of bax, fas, and p53 in the pathogenesis of early-stage mycosis fungoides and alterations in expression following photochemotherapy. Indian J Dermatol 2011;56:501504.

18. Fredholm S, Gjerdrum LM, Willerslev-Olsen A, Petersen DL, Nielsen IO, Kauczok CS, Wobser M, Ralfkiaer U, Bonefeld CM, Wasik MA, Krejsgaard T, Geisler C, Ralfkiaer E, Gniadecki R, Woetmann A, Odum N. STAT3 activation and infiltration of eosinophil granulocytes in mycosis fungoides. Anticancer Res 2014;34:5277-5286.

19. Mitteldorf C, Robson A, Tronnier M, Pfaltz MC, Kempf W. Galectin-3 Expression in Primary Cutaneous CD30-Positive Lymphoproliferative Disorders and Transformed Mycosis Fungoides. Dermatology 2015;231:164-170. 